\title{
Definition, diagnosis and management of fetal lower urinary tract obstruction: consensus of the ERKNet CAKUT-Obstructive Uropathy Work Group
}

Valentina Capone ${ }^{1 凶}{ }^{\square}$, Nicola Persico ${ }^{2}$, Alfredo Berrettini3 ${ }^{3}$, Stèphane Decramer ${ }^{4,5,6,7}$, Erika Adalgisa De Marco ${ }^{3}$, Diego De Palma ${ }^{8}$, Alessandra Familiari ${ }^{9}{ }^{9}$, Wout Feitz $\mathbb{1}^{10}$, Maria Herthelius (11) ${ }^{11}$, Vytis Kazlauskas ${ }^{12}$, Max Liebau (1) ${ }^{13}$, Gianantonio Manzoni ${ }^{3}{ }^{3}$, Michal Maternik $\mathbb{1}^{14}$, Giovanni Mosiello ${ }^{15}$, Joost Peter Schanstra ${ }^{4,5}$, Johan Vande Walle ${ }^{16}$, Elke Wüh/17, Elisa Ylinen ${ }^{18}$, Aleksandra Zurowska ${ }^{19}$, Franz Schaefer ${ }^{20}$ and

Giovanni Montini(iD ${ }^{1,21}$

Abstract | Fetal lower urinary tract obstruction (LUTO) is associated with high mortality and postnatal morbidity caused by lung hypoplasia and impaired kidney function. Specific diagnostic features that can guide clinical approach and decisions are lacking; thus, the European Reference Network for Rare Kidney Diseases established a work group to develop recommendations regarding the clinical definition, diagnosis and management of prenatally detected LUTO. The work group recommends the use of antero-posterior diameter of renal pelvis as the most reliable parameter for suspecting obstructive uropathies and for suspecting prenatal LUTO in the presence of fetal megacystis. Regarding prenatal and postnatal prognosis of fetuses with LUTO, the risk of fetal and neonatal death depends on the presence of oligohydramnios or anhydramnios before 20 weeks' gestation, whereas the risk of kidney replacement therapy cannot be reliably foreseen before birth. Parents of fetuses with LUTO must be referred to a tertiary obstetric centre with multidisciplinary expertise in prenatal and postnatal management of obstructive uropathies, and vesico-amniotic shunt placement should be offered in selected instances, as it increases perinatal survival of fetuses with LUTO.

凶e-mail: valentina.capone@ policlinico.mi.it

https://doi.org/10.1038/ s41585-022-00563-8
Fetal lower urinary tract obstruction (LUTO) affects $\sim 2-3$ out of 10,000 fetuses with a wide spectrum of severity ${ }^{1}$. LUTO diagnosis can be either prenatal or postnatal, with a 1:1 ratio, as described in a population-based study from 2005 (REF. ${ }^{1}$ ). Fetal LUTO aetiopathogenesis is related to an outflow obstruction of the bladder during fetal urinary tract development, which leads to progressive bladder dilation and bladder wall thickening, and subsequent hydro-ureteronephrosis, kidney parenchymal compression and reduced amniotic fluid production (oligohydramnios and anhydramnios) ${ }^{2,3}$. The reduction of amniotic fluid causes lung hypoplasia ${ }^{3}$, which can lead to fetal and perinatal mortality (combined mortality $60-80 \%$ when oligohydramnios or anhydramnios is diagnosed during the second trimester of gestation ${ }^{4,5}$ ) or to postnatal morbidity. Kidney hypodysplasia, another important complication of fetal LUTO, is part of the wide spectrum of congenital anomalies of the kidneys and of the urinary tract $^{6}$. Kidney damage in LUTO is primarily caused by the direct effect of urine flow obstruction on glomeruli development ${ }^{6}$. Acute urinary flow obstruction has been shown to cause a $50 \%$ loss of functioning nephrons after 6 days and a complete loss of nephrons after 6 weeks ${ }^{7}$. In this experimental setting of urinary flow obstruction, kidney damage is, therefore, secondary to a primary defect in the development of the entire urinary tract, which is caused by fetal urine flow obstruction and leads to a compression of kidney parenchyma ${ }^{2,3,8-10}$. Moreover, kidney hypodysplasia can be caused by mutations in genes known to have an important role in 
urinary tract and kidney parenchymal development ${ }^{11}$ A single mutation in one of those genes can, therefore, be responsible for both LUTO and kidney hypodysplasia ${ }^{11}$.

The high rate of termination of pregnancy, the various degrees of severity of the obstruction and the possibility of neonatal kidney replacement therapy all contribute to the difficulty in establishing the actual life expectancy in LUTO. Among patients who survive, the lifetime risk of developing end-stage kidney disease (ESKD) is $\sim 30 \%{ }^{12}$.

The most common causes of LUTO are posterior urethral valves (PUVs) and urethral atresia ${ }^{13}$; less common causes are anterior urethral valves, megalourethra, cloacal malformations and prolapsing ureterocele ${ }^{14}$. Typical sonographic features of fetuses with LUTO are enlarged bladder (megacystis) (FIG. 1), unilateral or bilateral hydronephrosis (FIG. 2), dilated ureters (FIG. 3) and oligohydramnios or anhydramnios ${ }^{8}$ (FIG. 3). Prenatal differential diagnosis of the cause of the LUTO can be challenging because different forms of LUTO present with similar findings on ultrasonography and, in up to one-third of suspected cases, a non-obstructive cause of the prenatal findings that are suggestive of LUTO (which is most often vesico-ureteral reflux) might be found at postnatal follow-up assessment ${ }^{8,15}$. However, the ability

\footnotetext{
Author addresses

'Pediatric Nephrology, Dialysis and Transplant Unit, Fondazione IRCCS Ca'

Granda-Ospedale Maggiore Policlinico, Milan, Italy.

${ }^{2}$ Fetal Medicine and Surgery Service, Fondazione IRCCS Ca' Granda, Ospedale Maggiore Policlinico, Milan, Italy.

'Pediatric Urology Unit, Fondazione IRCCS Ca' Granda-Ospedale Maggiore Policlinico, Milan, Italy.

${ }^{4}$ Institut National de la Santé et de la Recherche Médicale (INSERM), U1297, Institut of

Cardiovascular and Metabolic Disease, Toulouse, France.

5Université Toulouse III Paul-Sabatier, Toulouse, France.

${ }^{6}$ Service de Néphrologie Pédiatrique, Hôpital des Enfants, CHU Toulouse, Toulouse, France. ${ }^{7}$ Centre De Référence des Maladies Rénales Rares du Sud-Ouest (SORARE), Toulouse, France.

${ }^{8}$ Nuclear Medicine Unit, "Circolo" Hospital, ASST-settelaghi, Varese, Italy.

${ }^{9}$ High Risk Pregnancy Unit, Department of Women, Children and Public Health,

Fondazione Policlinico Universitario A. Gemelli IRCCS, Rome, Italy.

${ }^{10}$ Department of Urology, Division of Paediatric Urology, Radboudumc Amalia Children's

Hospital, Nijmegen, Netherlands.

${ }^{11}$ Karolinska Institutet and Karolinska University Hospital, Stockholm, Sweden.

${ }^{12} \mathrm{Clinic}$ of Gastroenterology, Nephrourology and Surgery, Institute of Clinical Medicine,

Faculty of Medicine, Vilnius University, Vilnius, Lithuania.

${ }^{13}$ Department of Pediatrics and Center for Molecular Medicine, Medical Faculty and

University Hospital Cologne, University of Cologne, Cologne, Germany.

${ }^{14}$ Department of Pediatrics, Nephrology and Hypertension, Medical University of Gdansk,

Gdansk, Poland.

${ }^{15}$ Bambino Gesù Pediatric Hospital, Division of Pediatric Urology/Neuro-Urology,

Rome, Italy.

${ }^{16}$ Paediatric Nephrology and Rheumatology Section, Department of Paediatrics,

Ghent University Hospital, Ghent, Belgium.

${ }^{17}$ Division of Pediatric Nephrology, Center for Pediatrics and Adolescent Medicine,

Heidelberg University Hospital, Heidelberg, Germany.

${ }^{18}$ Department of Pediatric Nephrology and Transplantation, New Children's Hospital,

University of Helsinki and Helsinki University Hospital, Helsinki, Finland.

${ }^{19}$ Centre of Rare Diseases, Medical University Gdansk, Gdansk, Poland.

${ }^{20}$ Division of Pediatric Nephrology, Center for Pediatrics and Adolescent Medicine,

University of Heidelberg, Heidelberg, Germany.

${ }^{21}$ Giuliana and Bernardo Caprotti Chair of Pediatrics, Department of Clinical Sciences

and Community Health, University of Milan, Milan, Italy.
}

to predict postnatal kidney function from fetal assessment is crucial to provide parents with adequate information on postnatal outcome, both in terms of mortality and risk of chronic kidney disease, and on the potential benefits and limitations of fetal intervention.

In 2018, the European Reference Network for Rare Kidney Diseases (ERKNet, which is endorsed and sponsored by the European Commission) established a work group to develop recommendations regarding the clinical definitions, diagnosis and management of prenatally detected LUTO, in collaboration with other European networks, in particular the European Reference Network on Urogenital Diseases and Conditions (ERN eUROGEN). Here, we present a Consensus Statement on the prenatal definition, diagnosis and management of obstructive uropathies, based on published information, as well as on the opinions of ERKNet and ERN eUROGEN experts. These recommendations are addressed to paediatric nephrologists, paediatric urologists and obstetricians dealing with prenatally suspected obstructive uropathies.

\section{Methods}

The Consensus Statement was developed following the Reporting Items for Practice Guidelines in Healthcare statement for practice guidelines ${ }^{16}$; the Delphi method ${ }^{17}$ was used to determine the extent of agreement and the areas of disagreement among the experts, in order to achieve a consensus opinion. The members of the ERKNet obstructive uropathy and kidney dysplasia work group and the experts on urinary tract obstructions from ERN eUROGEN formed the core work group, composed of eight paediatric nephrologists, four paediatric urologists, two fetal surgeons, one kidney biochemical researcher, one radiologist and one nuclear medicine physician. The core work group performed the literature search, discussed the main results and wrote the draft of the consensus paper. The first draft was discussed by the core group during a hybrid meeting held in February 2020 in Heidelberg and online (sponsored by the European Community through ERKNet), and was subsequently evaluated by a voting panel of $60 \mathrm{mem}$ bers from ERKNet, ERN eUROGEN and other networks with expertise in CAKUT, such as the European Society of Paediatric Nephrology and the Italian Society of Pediatric Nephrology. The voting panel was asked to provide a level of agreement with the recommendations from the core group using a four-point scale: strongly disagree, rather disagree, rather agree and strongly agree. Recommendations that did not reach a consensus of at least $75 \%$ of agreement (strongly plus rather agree) were discussed again by the working group, modified accordingly and reviewed again by the voting panel until a consensus level of at least $75 \%$ was achieved. The results of the present Consensus Statement will be periodically revised and updated by the group, possibly every 5 years.

\section{PICO questions}

Patients or Population covered, Intervention, Comparator, Outcome (PICO) questions were developed for the Consensus Statement ${ }^{18}$. The population of interest was fetuses with a suspicion of obstructive uropathy on ultrasonography. The intervention was prenatal treatment 


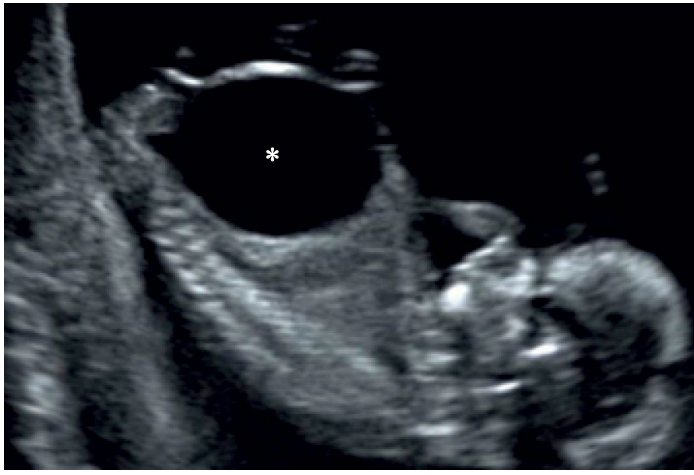

Fig. 1 | Fetal megacystis. Ultrasonography image of a 12-week-old fetus with megacystis, shown by the abnormally enlarged bladder (asterisk).

and the comparators were either no treatment or other treatment. The outcomes were kidney and fetal survival.

\section{Literature search}

The following key words were used to identify relevant studies published before the end of 2020: "urinary tract dilation", "urinary tract obstruction", "hydronephrosis", "hydroureteronephrosis", "megaureter", "posterior urethral valves", "ureterocele", "megacystis". The search retrieved 1,496 results. After excluding papers dealing with postnatal diseases, we retained 67 publications for analysis, 62 of which are referenced in the present paper, whereas 5 were excluded because they contained results already mentioned in other studies.

\section{Prenatal ultrasonography parameters to define urinary tract obstruction}

First, the work group discussed which prenatal ultrasonography parameters should be used to define urinary tract obstruction.

\section{The most reliable parameter for suspecting the presence of obstructive uropathy}

The presence of unilateral or bilateral hydronephrosis on prenatal fetal ultrasonography usually alerts the clinician to the possible presence of a congenital anomaly of the kidney and urinary tract ${ }^{19}$. Several grading systems have been proposed to stratify the severity of

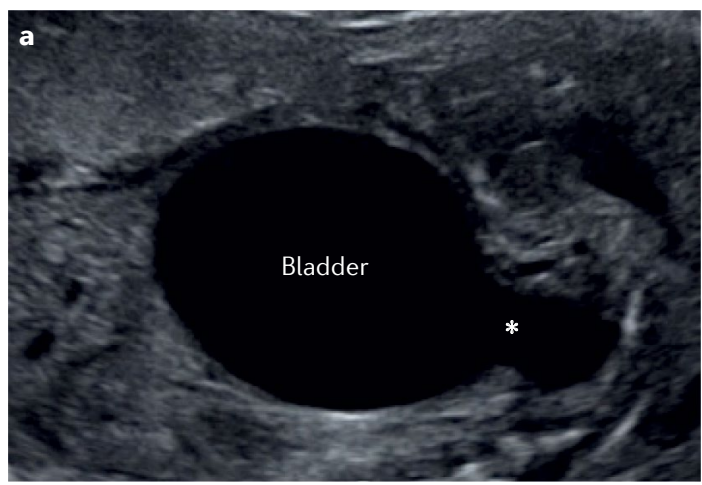

hydronephrosis in order to identify fetuses at high risk of morbidity who will need further imaging and possibly a prenatal or neonatal therapeutic procedure. Some of these grading systems are descriptive ${ }^{20}$, others quantitative (usually measuring the antero-posterior diameter (APD) of the renal pelvis) $)^{21}$ or semi-quantitative ${ }^{22,23}$, and different thresholds based on the trimester of pregnancy have been proposed ${ }^{24,25}$. Additional ultrasonography findings have been proposed to define and stratify the severity of prenatal urinary tract dilation (UTD), including the extent of calyceal dilation, the presence of parenchymal alterations (such as kidney hypoplasia and dysplasia), bladder and ureteral abnormalities, fetus gender and amniotic fluid volume (AFV) ${ }^{14}$. In 2014, eight scientific societies (The American College of Radiology, the American Institute of Ultrasound in Medicine, the American Society of Pediatric Nephrology, the Society for Fetal Urology, the Society for Maternal-Fetal Medicine, the Society for Pediatric Urology, the Society for Pediatric Radiology and the Society of Radiologists in Ultrasound) agreed to collaborate on the development of a unified grading system of prenatal and postnatal UTD, with the aim of selecting the most reliable parameters for a correct definition of UTD ${ }^{14}$. The scientific societies developed the UTD classification system, which stratifies the severity of UTD on the basis of gestational age (before and after 28 weeks' gestation) and of the most reliable sonographic parameters: APD of the renal pelvis; calyceal dilation; kidney parenchymal thickness and appearance; bladder and ureteral abnormalities. Pelvic dilation is defined by an APD of renal pelvis $\geq 7 \mathrm{~mm}$ at $16-27$ weeks' gestation and $\geq 10 \mathrm{~mm}$ at $\geq 28$ weeks. According to the classification system, low-grade APD (4-6 $\mathrm{mm}$ at $16-27$ weeks and $7-9 \mathrm{~mm}$ at $\geq 28$ weeks' gestation) should be given clinical consideration prenatally only if associated with one of the other radiological features mentioned ${ }^{14}$. Likewise, the presence of peripheral calyceal dilation alone should not be used for the definition of obstructive uropathy, but as an additional finding suggestive of a more severe obstruction ${ }^{14}$.

Recommendation 1. The most reliable parameter for suspecting the presence of obstructive uropathy is the antero-posterior diameter (APD) of the renal pelvis, as proposed by the Urinary Tract Dilatation (UTD)

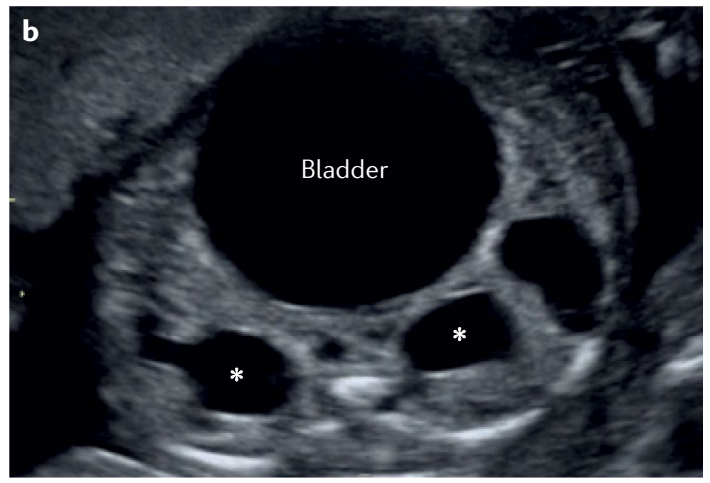

Fig. 2 | Fetal posterior urethral valves and hydronephrosis. Ultrasonography images of a 20-week-old fetus with posterior urethral valves, showing the typical findings of enlarged bladder with dilation of the bladder neck (the keyhole sign, asterisk in panel a) and bilateral hydronephrosis (asterisks in panel b). 


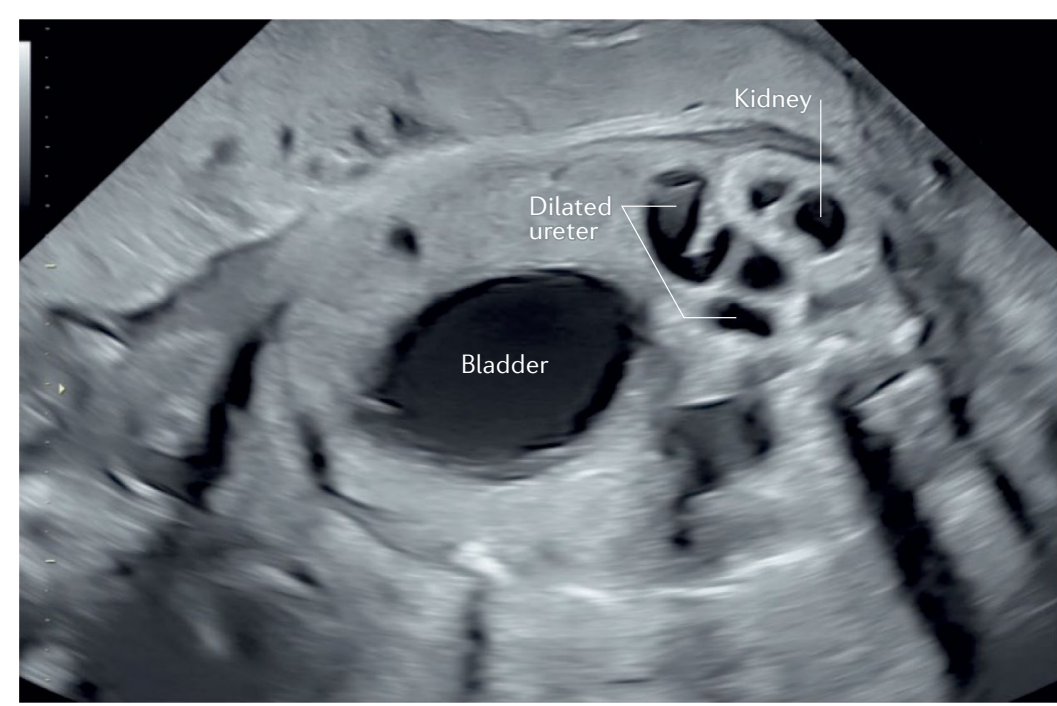

Fig. 3 | Fetal posterior urethral valves and anhydramnios. Ultrasonography image of a fetus with anhydramnios and a dilated ureter.

classification. Pelvic dilation is defined by an APD $\geq 7 \mathrm{~mm}$ at $16-27$ weeks and $\geq 10 \mathrm{~mm}$ at $\geq 28$ weeks' gestation. An APD of 4-6 mm at 16-27 weeks and 7-9 mm at $\geq 28$ weeks' gestation should be given clinical consideration prenatally only if associated with other signs of obstructive uropathy (BOX 1).

\section{Parameters for prenatal suspicion of LUTO}

The presence of an enlarged bladder (megacystis) is essential to distinguish lower from upper urinary tract obstructions. Megacystis in the first trimester of pregnancy is defined as a longitudinal bladder diameter $\geq 7 \mathrm{~mm}$ (FIG. 1). Longitudinal bladder diameter between $7 \mathrm{~mm}$ and $12 \mathrm{~mm}$ is often a transient finding that resolves in $\sim 90 \%$ of fetuses in the second trimester, whereas a measurement of $\geq 15 \mathrm{~mm}$ is strongly suggestive of LUTO, as resolution over the subsequent weeks of pregnancy is unlikely ${ }^{26}$. Evidence suggests that additional first trimester ultrasonography findings, such as nuchal translucency $>95^{\circ}$ percentile and umbilical cord cyst, might be useful in differentiating isolated PUV from complex megacystis (secondary to chromosomal abnormalities with multiple structural defects) and from urethral atresia, which is an unfavourable condition for antenatal treatment ${ }^{27}$. The finding of megacystis at any stage of pregnancy should prompt a detailed sonographic assessment of the fetal anatomy to exclude associated major extrarenal defects and to monitor the evolution of bladder enlargement ${ }^{27}$. Additionally, prenatal invasive testing (by chorionic villous sampling or amniocentesis with microarray analysis) or non-invasive prenatal testing (by prenatal cell-free DNA screening) should be offered when megacystis is found, to exclude associated chromosomal abnormalities ${ }^{27,28}$.

In the second trimester of pregnancy, megacystis is commonly defined subjectively as an enlarged bladder that fails to void over a period of $40 \mathrm{~min}$ during ultrasonographic examination, but no standardized measurement cut-offs defining the degree of bladder enlargement associated with obstruction are available ${ }^{29}$.
Prenatal LUTO has classically been suspected on the basis of three ultrasonographic findings: megacystis, dilated posterior urethra (known as the 'keyhole sign') ${ }^{21}$ and hydronephrosis ${ }^{8}$ (FIG. 2). A clinical score to differentiate LUTO from non-obstructive megacystis (mostly secondary to vesico-ureteral reflux, which can be diagnosed postnatally in $15-30 \%$ of prenatally suspected LUTO $^{8,15}$ ) during the second trimester of gestation has been developed on the basis of antenatal multivariate analysis of 143 fetuses with megacystis (defined as an enlarged bladder failing to empty during a 40 - min observation) diagnosed from the 18 th week onwards ${ }^{8}$. The score is based on five antenatal variables: bladder volume measurement $>35 \mathrm{~cm}^{3}$ or ascites at referral; bilateral ureteral dilation; reduced amniotic fluid (expressed as a single deepest pocket $<2 \mathrm{~cm}$ ); male fetal sex; and gestational age at referral $<28$ weeks. This score shows good discriminative capacity from non-obstructive megacystis (area under the curve $(\mathrm{AUC})=0.84, P<0.001$ ), and better diagnostic accuracy than the classic ultrasonography triad (megacystis, dilated posterior urethra and hydronephrosis) (AUC $=0.63, P=0.07)^{8}$.

Future studies are needed to validate these results, in order to adopt a reliable and reproducible quantitative ultrasonographic definition of megacystis during the second and third trimesters of pregnancy and to enable appropriate prenatal counselling and improved selection of candidates for fetal intervention.

In addition, selected instances, especially when ultrasonographic examination is difficult to perform because of oligohydramnios, can benefit from fetal magnetic resonance imaging, particularly after 20 weeks of gestation and when extrarenal manifestations have to be ruled out ${ }^{30}$.

Recommendation 2. Prenatal suspicion of LUTO requires the presence of an enlarged fetal bladder (megacystis) on obstetric ultrasound scan at any stage during pregnancy. In the first trimester, fetal megacystis (defined as a longitudinal bladder diameter of $\geq 7 \mathrm{~mm}$ ) is strongly suggestive of LUTO if the longitudinal bladder diameter is $\geq 15 \mathrm{~mm}$. Further research is needed to objectively define bladder enlargement during the second and third trimesters and to differentiate between obstructive and non-obstructive causes of prenatal megacystis through the development of a reliable severity scoring system (BOX 1).

\section{Survival and kidney outcome prediction in fetuses with LUTO}

The work group discussed the parameters of outcome prediction, in terms of survival and kidney function, in fetuses with LUTO.

\section{Outcome prediction}

The presence of oligohydramnios or anhydramnios (respectively defined as the deepest vertical pocket of amniotic fluid $\leq 2 \mathrm{~cm}$ and as complete absence of amniotic fluid) before 20 weeks gestation is a strong predictor of pulmonary hypoplasia and of fetal ${ }^{31-33}$ and neonatal death $^{34}$. Amniotic fluid acts as a protective cushion for the fetus and is essential for fetal lung development ${ }^{35}$. 
Fetal and perinatal survival of fetuses with oligohydramnios or anhydramnios is reported to be $15-24 \%$ and the high mortality is caused by lung hypoplasia and consequent pulmonary failure ${ }^{9,10}$. By contrast, the assessment of AFV as a predictor of postnatal kidney function is still debated ${ }^{19}$. Appearance on ultrasonography of the renal cortex (hyperechogenic parenchyma and/or presence of cysts) and oligohydramnios or anhydramnios have both shown limited performance as individual predictors of postnatal kidney failure in fetuses with LUTO, with a sensitivity and false-positive rate of $57 \%$ and $16 \%$, respectively, for renal cortical appearance, and of $63 \%$ and $24 \%$, respectively, for oligohydramnios ${ }^{36}$. A major drawback for the use of kidney echogenicity to assess fetal kidney function in the context of obstructive uropathy is the poor standardization of the definition and grading of echogenic kidneys, which might lead to substantial inter-observer variation in the interpretation of findings ${ }^{37}$. Several biomarkers from fetal urine, fetal blood and amniotic fluid have been investigated as potential predictors of postnatal kidney function in fetuses with

\section{Box 1 | ERKNet recommendations for prenatal definition, diagnosis and} management of LUTO

\section{Recommendation 1}

The most reliable parameter for suspecting the presence of obstructive uropathy is the antero-posterior diameter (APD) of the renal pelvis, as proposed by the Urinary Tract Dilation (UTD) classification. Pelvic dilation is defined by an APD $\geq 7 \mathrm{~mm}$ at $16-27$ weeks and $\geq 10 \mathrm{~mm}$ at $\geq 28$ weeks' gestation. An APD of $4-6 \mathrm{~mm}$ at $16-27$ weeks and $7-9 \mathrm{~mm}$ at $\geq 28$ weeks' gestation should be given clinical consideration prenatally only if associated with other signs of obstructive uropathy.

\section{Recommendation 2}

Prenatal suspicion of lower urinary tract obstruction (LUTO) requires the presence of an enlarged fetal bladder (megacystis) on obstetric ultrasound scan at any stage during pregnancy. In the first trimester, fetal megacystis (defined as a longitudinal bladder diameter of $\geq 7 \mathrm{~mm}$ ) is strongly suggestive of LUTO if the longitudinal bladder diameter is $\geq 15 \mathrm{~mm}$. Further research is needed to objectively define bladder enlargement during the second and third trimesters and to differentiate between obstructive and non-obstructive causes of prenatal megacystis through the development of a reliable severity scoring system.

\section{Recommendation 3}

The risk of fetal and neonatal death has to be based on the presence of oligohydramnios or anhydramnios before 20 weeks' gestation, which is a strong predictor of pulmonary hypoplasia.

\section{Recommendation 4}

The risk of kidney replacement therapy cannot be foreseen before birth, as amniotic fluid volume, kidney parenchymal echogenicity and fetal urine biomarkers are not reliable predictors. Normal postnatal kidney function is also not predictable by a normal amount of amniotic fluid.

\section{Recommendation 5}

Multi-parameter scoring systems to stage the severity of LUTO and to predict postnatal outcome have been proposed, but have not yet been clinically adopted. Further clinical validation and the adoption of standardized assessment across prenatal centres are needed.

\section{Recommendation 6}

Fetuses with prenatal LUTO must be referred to a tertiary obstetric centre with multidisciplinary expertise in prenatal and postnatal management of obstructive uropathies.

\section{Recommendation 7}

Based on existing evidence that vesico-amniotic shunt placement increases perinatal survival in fetuses with LUTO, fetal intervention should be offered in selected cases. However, parents should be made aware of the residual risk of long-term mortality and kidney function impairment.

ERKNet, European Reference Network for Rare Kidney Diseases.
LUTO $^{38-40}$. Fetal blood and/or urine biomarkers including sodium, chloride, calcium, osmolarity and low molecular weight proteins, such as $\beta_{2}$-microglobulin $\left(\beta_{2} \mathrm{~m}\right)$, have been proposed as predictors of postnatal kidney function in fetuses with LUTO $^{38,39}$. However, none of these biomarkers is able to reliably predict postnatal kidney function ${ }^{40}$. A particular effort has been invested in measuring fetal urine and blood levels of $\beta_{2} \mathrm{~m}$, a protein that, unlike creatinine, does not cross the placenta and is not cleared by the mother ${ }^{41}$, theoretically providing a good marker of fetal kidney function; results from studies investigating $\beta_{2} \mathrm{~m}$ levels in fetal serum showed good sensitivity $(80-100 \%)$ and specificity (66-99\%) of $\beta_{2} \mathrm{~m}$ in predicting postnatal kidney function $^{42-45}$. In particular, fetal serum $\beta_{2}$ m levels measured late in gestation (week 32) displayed high sensitivity $(96 \%)$ and specificity ( $86 \%)$ in predicting postnatal kidney function ${ }^{43}$. Fetal urine $\beta_{2} \mathrm{~m}$ levels showed correlation with a decreased number of glomeruli in fetuses with obstructive uropathy ${ }^{44}$. However, variable $\beta_{2}$ m cut-off values were used in these studies, and a large-scale validation study exploring the optimal $\beta_{2}$ m cut-off for both fetal blood and urine values is needed. A novel approach to predicting postnatal kidney function is proteome analysis of fetal urine in order to identify a spectrum of peptides able to predict postnatal kidney function ${ }^{46,47}$. Proteomics analysis of fetal urine in fetuses with PUV (used as the prototypic LUTO) ${ }^{46}$ showed a 12-peptide signature (12PUV), which is specifically expressed in fetuses who would develop ESKD before the age of 2 years ${ }^{46}$. These results have been validated in an independent, blinded cohort of 38 patients with $\mathrm{PUV}^{46}$ and in an independent, small, single-centre validation study of 14 fetuses with $\mathrm{PUV}^{47}$. In the first study, the 12PUV signature showed high sensitivity $(88 \%)$ and specificity (95\%) in predicting the occurrence of ESKD before the age of 2 years ${ }^{46}$, whereas, in the second study, the 12PUV signature showed high accuracy $90 \%$ (95\% CI $78-100$ ) in predicting kidney failure (defined by an estimated glomerular filtration rate $<60 \mathrm{ml} / \mathrm{min} / 1.73 \mathrm{~m}^{2}$ ) at 6 months $^{47}$. Interestingly, in both studies ${ }^{46,47}$, the $12 \mathrm{PUV}$ signature outperformed ultrasonography features such as AFV and kidney parenchymal appearance (presence of cysts or increased parenchymal echogenicity). The signature also predicted postnatal kidney function better than fetal urine $\beta_{2} \mathrm{~m}$ levels ${ }^{46}$. To further refine the molecular signature of PUV, fetal urinary metabolites were analysed in two populations of fetuses with PUV (group 1: 13 fetuses who developed ESKD at 2 years postnatally; group 2: 12 fetuses who did not develop ESKD at 2 years postnatally ${ }^{48}$, identifying 24 metabolites highly expressed in group 1 only. The combination of metabolomics (24 metabolites) and peptidomics (12PUV) was then validated in an independent cohort of 35 fetuses with PUV, showing better accuracy than metabolomics alone ( $86 \%$ versus $60 \%, P=0.002$ ) but not of peptdidomics alone ( $86 \%$ versus $80 \%, P=0.344$ ) in predicting ESKD at the age of 2 years ${ }^{48}$. The final validation of the 12PUV signature is currently ongoing in the first clinical proteomics study in prenatal medicine (NCT03116217 $\left(\right.$ REF. $\left.^{49}\right)$ ), which aims to include 400 patients with PUV. In conclusion, fetal biomarkers showed promising results 
and can potentially complement clinical evaluation in LUTO, but require more extensive validation before being applied in clinical practice.

Recommendation 3. The risk of fetal and neonatal death has to be based on the presence of oligohydramnios or anhydramnios before 20 weeks' gestation, which is a strong predictor of pulmonary hypoplasia (BOX 1).

Recommendation 4. The risk of kidney replacement therapy cannot be foreseen before birth, as amniotic fluid volume, kidney parenchymal echogenicity and fetal urine biomarkers are not reliable predictors. Normal postnatal kidney function is also not predictable by a normal amount of amniotic fluid (BOX 1)

\section{Staging}

In total, two staging systems have been proposed to grade the severity of prenatal LUTO in relation to postnatal survival and residual kidney function ${ }^{50,51}$ (TABLE 1). In the first staging system ${ }^{50}$, LUTO is graded using several parameters, such as AFV, kidney echogenicity, cortical cysts, kidney dysplasia and fetal urinary biochemistry. According to this staging, stage I (or mild LUTO) presents with normal amniotic fluid levels from 18 weeks' gestation onwards and normal fetal kidney function defined as no evidence of kidney dysplasia, kidney cysts and normal fetal urine biochemistry; stage II (or moderate LUTO) presents with oligohydramnios or anhydramnios and normal fetal kidney function; stage III (or severe LUTO) presents with oligohydramnios or anhydramnios along with abnormal fetal kidney function, defined as kidney dysplasia and/or cysts and abnormal fetal urine biochemistry. In the study introducing this staging system $^{50}$, two fetuses with stage I LUTO survived with normal kidney function (with serum creatinine $<0.5 \mathrm{mg} / \mathrm{dl}$ at 6 months of age $)^{50}$. Fetal vesico-amniotic shunt (VAS) placement was done in 14/16 moderate instances of LUTO (stage II), and survival was $75 \%$, with $33 \%$ of ESKD at 6 months ${ }^{50}$; among the 7 fetuses with stage III LUTO, who were not offered any fetal intervention, only 1 survived, with a need for kidney replacement therapy ${ }^{50}$. These results suggest that patients with stage II LUTO are the ones who are most likely to benefit from fetal intervention, both in terms of survival and prevention of kidney damage ${ }^{50}$. The second staging system for LUTO ${ }^{51}$
(TABLE 1) is simple and is based on the combination of bladder volume (measured using ultrasonography) and gestational age at first onset of oligohydramnios. In this study, fetuses with normal AFV at 26 weeks' gestation have low postnatal mortality (9\%) and are classified as having mild $\mathrm{LUTO}^{51}$. Fetuses with bladder volume $\geq 5.4 \mathrm{~cm}^{3}$ and/or oligohydramnios or anhydramnios at 20 weeks' gestation have increased perinatal mortality $(55 \%)$ and are classified as severe $\mathrm{LUTO}^{51}$. All the other instances with bladder volume $<5.4 \mathrm{~cm}^{3}$ and/or normal AFV at 20 weeks' gestation are classified as moderate LUTO, with a perinatal mortality of $26 \%{ }^{51}$. Similar to perinatal mortality, severe impairment of kidney function (assessed by estimated glomerular filtration rate $<30 \mathrm{ml} / \mathrm{min} / 1.73 \mathrm{~m}^{2}$ ) was also shown to correlate with LUTO severity, and was reported in $11 \%$ of 36 fetuses with mild LUTO, $31 \%$ of 16 fetuses with moderate LUTO and $44 \%$ of 9 fetuses with severe LUTO $^{51}$.

A general agreement on the importance of staging the severity of LUTO to improve the prediction of postnatal outcome and to select patients who would benefit from fetal surgical intervention has been reached, but the current evidence is still insufficient to recommend one staging system over the other. Moreover, the first staging system is difficult to apply in clinical practice as it requires three consecutive fetal urine collections. An international consensus aiming to define a standard set of prenatal parameters for severity staging of LUTO and to agree on the type, frequency and duration of postnatal follow-up monitoring is urgently needed. This approach would enable a more meaningful comparison between fetal intervention and conservative management results across different studies than can currently be performed.

Recommendation 5. Multi-parameter scoring systems to stage the severity of LUTO and to predict postnatal outcome have been proposed, but have not yet been clinically adopted. Further clinical validation and the adoption of standardized assessment across prenatal centres are needed (BOX 1).

\section{Indications, timing and outcome of fetal surgical intervention in fetuses with LUTO}

The work group revised the indications and benefits of fetal surgical intervention in fetuses with LUTO.

Table 1 | Proposed classifications of fetal lower urinary tract obstruction according to severity

\begin{tabular}{|lllll|}
$\begin{array}{l}\text { Total } \\
\text { number } \\
\text { of fetuses }\end{array}$ & $\begin{array}{l}\text { LUTO severity stage } \\
\text { Stage I/mild }\end{array}$ & Stage II/moderate & Stage III/severe & Ref. \\
\hline 25 & $\begin{array}{l}\text { Normal AFV, normal } \\
\text { echogenicity of fetal } \\
\text { kidneys, no renal cysts, no } \\
\text { renal dysplasia, favourable } \\
\text { fetal urinary biochemistry } \\
\text { (n=2 fetuses) }\end{array}$ & $\begin{array}{l}\text { Oligohydramnios or } \\
\text { anhydramnios, hyperechogenic } \\
\text { kidneys, no renal cysts, no renal } \\
\text { dysplasia, favourable urinary } \\
\text { biochemistry (on 3 consecutive } \\
\text { evaluations) }(n=16 \text { fetuses) }\end{array}$ & $\begin{array}{l}\text { Oligohydramnios (but usually } \\
\text { anhydramnios), hyperechogenic } \\
\text { kidneys, possible renal cysts, possible } \\
\text { biochemistry (on 3 consecutive } \\
\text { evaluations) ( } n=7 \text { fetuses) }\end{array}$ \\
\hline 141 & $\begin{array}{l}\text { Normal AFV at 26 weeks' } \\
\text { gestation ( } n=70 \text { fetuses) }\end{array}$ & $\begin{array}{l}\text { Bladder volume }<5.4 \mathrm{~cm}^{3} \\
\text { and/or normal AFV at 20 weeks } \\
(n=38 \text { fetuses) }\end{array}$ & $\begin{array}{l}\text { Bladder volume } \geq 5.4 \mathrm{~cm}^{3} \text { and/or } \\
\text { oligohydramnios or anhydramnios } \\
\text { before 20 weeks' gestation ( } n=33 \\
\text { fetuses) }\end{array}$ & 51 \\
\hline
\end{tabular}

AFV, amniotic fluid volume; LUTO, lower urinary tract obstruction. 
Fetal intervention: vesico-amniotic shunt placement Potential outcomes of fetal LUTO include fetal and neonatal mortality, preterm delivery, poor postnatal kidney function, neonatal dialysis requirement, poor postnatal pulmonary function, prolonged mechanical ventilation and long-term bladder dysfunction ${ }^{15}$. The presence of anhydramnios early in the second trimester results in a fetal mortality rate close to $100 \%{ }^{31-33}$ and untreated fetal LUTO can lead to death in up to $80 \%$ of fetuses ${ }^{4,5}$, as this gestational period (canalicular phase) is crucial for lung development ${ }^{52,53}$.

LUTO is usually diagnosed at 20 weeks' gestation, when a detailed fetal anomaly scan is performed ${ }^{52}$. VAS placement is the most common prenatal intervention in fetuses with LUTO and consists of placing a shunt into the bladder of the fetus to enable continuous drainage into the amniotic cavity and bypass the blockage, to decompress the obstructed fetal bladder and restore AFV, preventing oligohydramnios and pulmonary hypoplasia $^{54}$ (FIG. 4). Procedure-related complications after VAS placement can occur in $\sim 40 \%$ of patients and include shunt dislocation or retraction, shunt migration, shunt blockage, fetal ascites, premature rupture of membranes, preterm labour, shunt site abdominal wall herniation and, in some instances, fetal demise $\mathrm{e}^{52,54-56}$.

The challenge after LUTO diagnosis is deciding whether or not VAS placement is needed ${ }^{57}$. The currently available data used to guide clinicians and parents in the decision include one randomized controlled trial (the PLUTO Trial) ${ }^{54}$, one prospective registry ${ }^{56}$ and numerous single-institution retrospective series included in a meta-analysis ${ }^{58}$. All the available studies are burdened by selection biases and the number of patients is still relatively limited, with high heterogeneity in the method of assessing postnatal kidney function ${ }^{52,55-58}$. In particular, the PLUTO trial, which aimed to compare VAS placement with the conservative approach, only recruited 31 patients because of the high number of pregnant women who did not want to receive prenatal VAS, resulting in unpowered conclusions ${ }^{54}$.

In 2017 , a meta-analysis was performed including 246 fetuses from 9 large datasets (including the PLUTO trial) ${ }^{52}$, who either underwent VAS placement between 20 and 27 weeks' gestation or were treated conservatively with amnio-infusions or simply received ultrasonography, with up to 2 years' postnatal followupmonitoring ${ }^{58}$. Results from this analysis showed that VAS placement improved perinatal survival, defined as survival up to 6 months of age (57\% in fetuses with VAS versus $39 \%$ in fetuses managed conservatively, $P<0.01$, OR 2.54), but did not affect survival at 6-12 months in four studies (44.2\% in fetuses with VAS versus $41.8 \%$ in fetuses managed conservatively) or at 2 years in three studies $(40.0 \%$ in fetuses with VAS versus $44.6 \%$ in fetuses managed with conservative treatment $)^{58}$. Moreover, in three studies with 2-year follow-up data, VAS did not improve postnatal kidney function, with abnormal kidney function reported in $47 \%$ of children $^{58}$.

In conclusion, it seems that VAS placement between 20 and 27 weeks' gestation is associated with a higher perinatal survival than expectant management, although

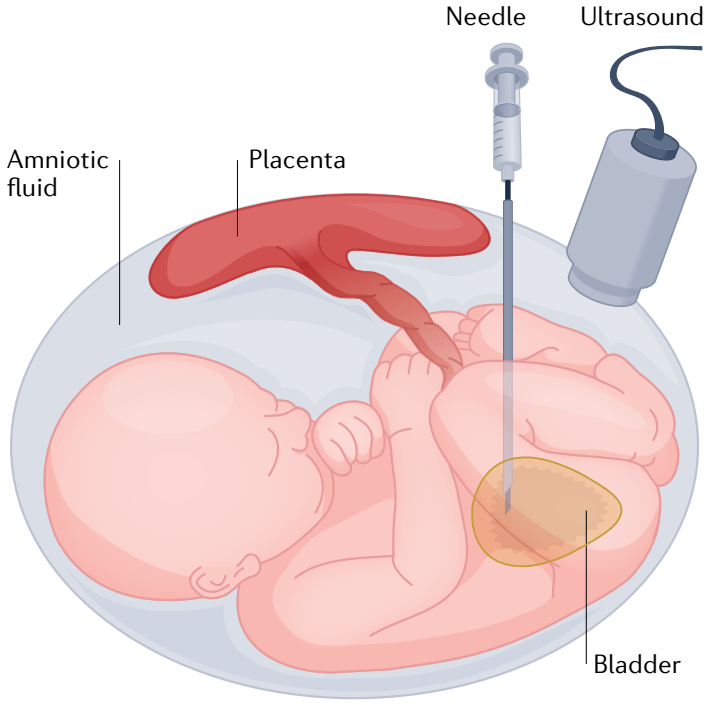

Fig. 4 | Prenatal vesico-amniotic shunt placement. A fetus with megacystis undergoes vesico-amniotic shunt placement under ultrasound guidance. A trocar (needle) is introduced through the mother's abdominal and uterine wall into the amniotic cavity and then into the bladder of the fetus. A drainage catheter is introduced through the trocar, with one end in the bladder and the other in the amniotic cavity. The correct position of the catheter is confirmed by ultrasonography. Adapted from REF ${ }^{63}$, Springer Nature Limited.

benefits for 1-year survival and for kidney function are currently uncertain ${ }^{58}$.

A useful tool for selecting patients who could most benefit from prenatal surgical treatment could be the three-stage classification system of LUTO (stage I, II, and III) $)^{50}$, based on which, patients with stage II LUTO (characterized by oligohydramnios or anhydramnios, hyperechogenic kidneys but absent renal cortical cysts or apparent signs of kidney dysplasia and favourable fetal urinary biochemistry) would most benefit from prenatal treatment of LUTO to prevent pulmonary hypoplasia and kidney failure ${ }^{50}$. In stage III LUTO (characterized by oligohydramnios or anhydramnios with hyperechogenic kidneys, cortical cysts and kidney dysplasia, as well as unfavourable fetal urinary biochemistry after serial evaluation), fetal intervention could prevent severe pulmonary hypoplasia but not kidney failure ${ }^{50,59}$.

Nevertheless, the available severity scoring systems are either based on very few patients or have not yet been validated; thus, we suggest careful and cautious use by clinicians, to select patients who can most benefit from prenatal intervention.

Fetal intervention: cystoscopic laser ablation of PUVs Fetal cystoscopic laser ablation of PUVs, consisting of the identification and fulguration of the PUV with a diode laser through fetal cystoscopy under local anaesthesia, has been proposed as an alternative to $\mathrm{VAS}^{60}$. A 2011 systematic review of existing literature reporting on fetal cystoscopy in LUTO retrieved four papers describing 63 fetuses treated with either fetal cystoscopy or VAS placement, or expectant management ${ }^{60}$. 
The review concluded that fetal cystoscopy laser valve ablation improved perinatal survival (OR 20.51, 95\% CI 3.87-108.69) compared with no treatment, but no differences in perinatal survival were observed between fetal cystoscopy and VAS placement (OR 1.49, 95\% CI $0.13-16.97)^{60}$. In addition, urological fistulas have been reported in $\sim 10 \%$ of fetuses undergoing cystoscopic laser ablation ${ }^{61}$, probably owing to the difficulty in directing the laser energy in alignment with the urethral canal in some instances, depending mostly on fetal and placental position $^{61}$. Technological improvement and development of new devices that will enable improved flexibility in visualizing the urethral outlet and directing the laser energy might reduce the occurrence of this type of complication ${ }^{62}$.

In conclusion, fetuses with LUTO should be evaluated by a multidisciplinary team consisting of a gynaecologist, a neonatologist, a paediatric nephrologist and a paediatric urologist, to select the fetuses who could most benefit from prenatal intervention, according to the available staging systems. When the multidisciplinary team decides that prenatal intervention is indicated, we recommend VAS placement, ideally performed before 27 weeks' gestation.

Recommendation 6. Fetuses with prenatal LUTO must be referred to a tertiary obstetric centre with multidisciplinary expertise in prenatal and postnatal management of obstructive uropathies (BOX 1)
Recommendation 7. Based on existing evidence that vesico-amniotic shunt placement increases perinatal survival in fetuses with LUTO, fetal intervention should be offered in selected cases. However, parents should be made aware of the residual risk of long-term mortality and kidney function impairment (BOX 1).

\section{Conclusions}

Prenatal diagnosis, severity assessment and correct management of LUTO are challenging, given the lack of specific diagnostic features that can guide clinical approaches and decisions. Fetuses with prenatal megacystis need to be referred to a tertiary obstetric centre with multidisciplinary expertise in prenatal and postnatal management of obstructive uropathies. If prenatal treatment is indicated, parents must be informed about the potential benefits for postnatal survival, but they should also be made aware of the residual risk of postnatal kidney failure, despite fetal intervention. Further studies are needed to improve standardization of ultrasonographic diagnostic parameters, validate prenatal biomarkers and improve staging systems to optimize prenatal care and to provide a more accurate selection of patients who might benefit most from fetal intervention, in terms of long-term survival and kidney function.

Published online 8 February 2022
1. Anumba, D. O., Scott, J. E., Plant, N. D. \& Robson, S. C. Diagnosis and outcome of fetal lower urinary tract obstruction in the northern region of England. Prenat. Diagn. 25, 7-13 (2005).

2. Morris, R. K. \& Kilby, M. D. Congenital urinary tract obstruction. Best Pract. Res. Clin. Obstet. Gynaecol. 22, 97-122 (2008)

3. Farrugia, M. K. Fetal bladder outlet obstruction embryopathology, in utero intervention and outcome. J. Pediatr. Urol. 12, 296-303 (2016).

4. Hobbins, J. C. et al. Antenatal diagnosis of renal anomalies with ultrasound. I. Obstructive uropathy. Am. J. Obstet. Gynecol. 148, 868-877 (1984).

5. Reuss, A., Wladimiroff, J. W., Stewart, P. A. \& Scholtmeijer, R. J. Non-invasive management of fetal obstructive uropathy. Lancet 2, 949-951 (1988).

6. Matsell, D. G., Mok, A. \& Tarantal, A. F. Altered primate glomerular development due to in utero urinary tract obstruction. Kidney Int. 61, 1263-1269 (2002).

7. Gough, D. in Essentials of Paediatric Urology (eds Rickwood, A. M. K., Thomas, D. F. M. \& Duffy P. G.) (Martin Dunitz Ltd, 2002).

8. Fontanella, F. et al. Prenatal diagnosis of LUTO: improving diagnostic accuracy. Ultrasound Obstet. Gynecol. 52, 739-743 (2018).

9. Grijseels, E. W. et al. Outcome of pregnancies complicated by oligohydramnios or anhydramnios of renal origin. Prenat. Diagn. 31, 1039-1045 (2011).

10. Melo, B. F. et al. Early risk factors for neonatal mortality in CAKUT: analysis of 524 affected newborns. Pediatr. Nephrol. 27, 965-972 (2012).

11. Capone, V. P., Morello, W., Taroni, F. \& Montini, G. Genetics of congenital anomalies of the kidney and urinary tract: the current state of play. Int. J. Mol. Sci. 18, 796 (2017).

12. Heikkila, J., Holmberg, C., Kyllonen, L., Rintala, R. \& Taskinen, S. Long-term risk of end stage renal disease in patients with posterior urethral valves. J. Urol. 186 , 2392-2396 (2011)

13. Ruano, R. et al. Fetal intervention for severe lower urinary tract obstruction: a multicenter case-control study comparing fetal cystoscopy with vesicoamniotic shunting. Ultrasound Obstet. Gynecol. 45, 452-458 (2015).

14. Nguyen, H. T. et al. Multidisciplinary consensus on the classification of prenatal and postnatal urinary tract dilation (UTD classification system). J. Pediatr. Urol. 10, 982-998 (2014).

15. Malin, G., Tonks, A. M., Morris, R. K., Gardosi, J. \& Kilby, M. D. Congenital lower urinary tract obstruction: a population-based epidemiological study. BJOG 119 , 1455-1464 (2012).

16. Chen, Y. et al. A reporting tool for practice guidelines in health care: the RIGHT Statement. Ann. Intern. Med. 166, 128-132 (2017)

17. de Villiers, M. R., de Villiers, P. J. \& Kent, A. P. The Delphi technique in health sciences education research. Med. Teach. 27, 639-643 (2005).

18. Guyatt, G. H. et al. GRADE guidelines: 2. Framing the question and deciding on important outcomes. J. Clin Epidemiol. 64, 395-400 (2011).

19. Aulbert, W. \& Kemper, M. J. Severe antenatally diagnosed renal disorders: background, prognosis and practical approach. Pediatr. Nephrol. 31, 563-574 (2016).

20. Ellenbogen, P. H., Scheible, F. W., Talner, L. B. $\delta$ Leopold, G. R. Sensitivity of gray scale ultrasound in detecting urinary tract obstruction. Am. J. Roentgenol. 130, 731-733 (1978).

21. Grignon, A. et al. Urinary tract dilatation in utero: classification and clinical applications. Radiology 160 645-647 (1986)

22. Riccabona, $\mathbf{M}$. et al. Imaging recommendations in paediatric uroradiology: minutes of the ESPR workgroup session on urinary tract infection, fetal hydronephrosis, urinary tract ultrasonography and voiding cystourethrography, Barcelona, Spain, June 2007. Pediatr. Radiol. 38, 138-145 (2008)

23. Onen, A. An alternative grading system to refine the criteria for severity of hydronephrosis and optimal treatment guidelines in neonates with primary UPJ-type hydronephrosis. J. Pediatr. Urol. 3, 200-205 (2007).

24. Odibo, A. O. et al. Mild pyelectasis: evaluating the relationship between gestational age and renal pelvic anterior-posterior diameter. Prenat. Diagn. 23, 824-827 (2003)

25. Chitty, L. S. \& Altman, D. G. Charts of fetal size: kidney and renal pelvis measurements. Prenat. Diagn. 23, 891-897 (2003)

26. Sebire, N. J., Von Kaisenberg, C., Rubio, C. Snijders, R. J. \& Nicolaides, K. H. Fetal megacystis at $10-14$ weeks of gestation. Ultrasound Obstet. Gynecol. 8, 387-390 (1996).
27. Fontanella, F. et al. Antenatal workup of early megacystis and selection of candidates for fetal therapy. Fetal Diagn. Ther 45, 155-161 (2019).

28. Ibirogba, E. R., Haeri, S. \& Ruano, R. Fetal lower urinary tract obstruction: what should we tell the prospective parents? Prenat. Diagn. 40, 661-668 (2020).

29. Fontanella, F. et al. Fetal megacystis: a lot more than LUTO. Ultrasound Obstet. Gynecol. 53, 779-787 (2019).

30. Fazecas, T. M. et al. Applicability of magnetic resonance imaging in the assessment of fetal urinary tract malformations. Can. Assoc. Radiol. J. 70, 83-95 (2019).

31. Shipp, T. D., Bromley, B., Pauker, S., Frigoletto, F. D. Jr. \& Benacerraf, B. R. Outcome of singleton pregnancies with severe oligohydramnios in the second and third trimesters. Ultrasound Obstet. Gynecol. 7, 108-113 (1996).

32. Mercer, L. J. \& Brown, L. G. Fetal outcome with oligohydramnios in the second trimester. Obstet Gynecol. 67, 840-842 (1986).

33. Barss, V. A., Benacerraf, B. R. \& Frigoletto, F. D. Jr. Second trimester oligohydramnios, a predictor of poor fetal outcome. Obstet. Gynecol. 64, 608-610 (1984).

34. Moxey-Mims, M. \& Raju, T. N. K. Anhydramnios in the setting of renal malformations: the National Institutes of Health Workshop Summary. Obstet. Gynecol. 131 1069-1079 (2018).

35. Underwood, M. A., Gilbert, W. M. \& Sherman, M. P. Amniotic fluid: not just fetal urine anymore. J. Perinatol. 25, 341-348 (2005)

36. Morris, R. K., Malin, G. L., Khan, K. S. \& Kilby, M. D. Antenatal ultrasound to predict postnatal renal function in congenital lower urinary tract obstruction: systematic review of test accuracy. BJOG 116, 1290-1299 (2009).

37. Hindryckx, A. \& De Catte, L. Prenatal diagnosis of congenital renal and urinary tract malformations. Facts Views Vis. Obgyn. 3, 165-174 (2011).

38. Elder, J. S., O'Grady, J. P., Ashmead, G., Duckett, J. W. $\&$ Philipson, E. Evaluation of fetal renal function: unreliability of fetal urinary electrolytes. J. Urol. 144, 574-578; discussion 593-594 (1990).

39. Glick, P. L. et al. Management of the fetus with congenital hydronephrosis II: prognostic criteria and selection for treatment. J. Pediatr. Surg. 20, 376-387 (1985). 
40. Morris, R. K., Quinlan-Jones, E., Kilby, M. D. \& Khan, K. S. Systematic review of accuracy of fetal urine analysis to predict poor postnatal renal function in cases of congenital urinary tract obstruction. Prenat. Diagn. 27, 900-911 (2007).

41. Berry, S. M. et al. Predictive value of fetal serum beta 2-microglobulin for neonatal renal function. Lancet 345, 1277-1278 (1995).

42. Spaggiari, E. et al. Fetal obstructive uropathy complicated by urinary ascites: outcome and prognostic value of fetal serum beta-2-microglobulin Ultrasound Obstet. Gynecol. 41, 185-189 (2013).

43. Spaggiari, E. et al. Sequential fetal serum beta2microglobulin to predict postnatal renal function in bilateral or low urinary tract obstruction. Ultrasound Obstet. Gynecol. 49, 617-622 (2017).

44. Luton, D. et al. Foetal serum but not urinary beta2 microglobulin correlates with histological injury to the kidney. Clin. Biochem. 46, 1607-1610 (2013).

45. Dommergues, M. et al. Fetal serum beta2microglobulin predicts postnatal renal function in bilateral uropathies. Kidney Int. 58, 312-316 (2000).

46. Klein, J. et al. Fetal urinary peptides to predict postnatal outcome of renal disease in fetuses with posterior urethral valves (PUV). Sci. Transl. Med. 5, 198ra106 (2013)

47. Buffin-Meyer, B. et al. A single-center study to evaluate the efficacy of a fetal urine peptide signature predicting postnatal renal outcome in fetuses with posterior urethral valves. Pediatr. Nephrol. 35 , 469-475 (2020)

48. Buffin-Meyer, B. et al. Combination of the fetal urinary metabolome and peptidome for the prediction of postnatal renal outcome in fetuses with PUV. J. Proteom. 184, 1-9 (2018).

49. Buffin-Meyer, B. et al. The ANTENATAL multicentre study to predict postnatal renal outcome in fetuses with posterior urethral valves: objectives and design Clin. Kidney J. 13, 371-379 (2020).

50. Ruano, R. et al. Fetal lower urinary tract obstruction proposal for standardized multidisciplinary prenatal management based on disease severity. Ultrasound Obstet. Gynecol. 48, 476-482 (2016).

51. Fontanella, F. et al. Antenatal staging of congenital lower urinary tract obstruction. Ultrasound Obstet. Gynecol. 53, 520-524 (2019).

52. Morris, R. K et al. The Percutaneous shunting in Lower Urinary Tract Obstruction (PLUTO) study and randomised controlled trial: evaluation of the effectiveness, cost-effectiveness and acceptability of percutaneous vesicoamniotic shunting for lower urinary tract obstruction. Health Technol. Assess. 17. $1-232$ (2013).

53. Williams, O., Hutchings, G., Hubinont, C., Debauche, C. $\&$ Greenough, A. Pulmonary effects of prolonged oligohydramnios following mid-trimester rupture of the membranes-antenatal and postnatal management. Neonatology 101, 83-90 (2012).

54. Morris, R. K. et al. Percutaneous vesicoamniotic shunting versus conservative management for fetal lower urinary tract obstruction (PLUTO): a randomised trial. Lancet 382, 1496-1506 (2013)

55. Morris, R. K., Malin, G. L., Khan, K. S. \& Kilby, M. D. Systematic review of the effectiveness of antenatal intervention for the treatment of congenital lower urinary tract obstruction. BJOG 117, 382-390 (2010)

56. Morris, R. K. et al. Outcome in fetal lower urinary tract obstruction: a prospective registry study. Ultrasound Obstet. Gynecol. 46, 424-431 (2015).

57. Biard, J. M. et al. Long-term outcomes in children treated by prenatal vesicoamniotic shunting for lower urinary tract obstruction. Obstet. Gynecol. 106, 503-508 (2005).

58. Nassr, A. A. et al. Effectiveness of vesicoamniotic shunt in fetuses with congenital lower urinary tract obstruction an updated systematic review and meta-analysis. Ultrasound Obstet. Gynecol. 49, 696-703 (2017).

59. Ruano, R Dunn, T. Braun, M. C. Angelo, J. R. $\&$ Safdar, A. Lower urinary tract obstruction: fetal intervention based on prenatal staging. Pediatr. Nephrol. 32, 1871-1878 (2017)

60. Morris, R. K., Ruano, R. \& Kilby, M. D. Effectiveness of fetal cystoscopy as a diagnostic and therapeutic intervention for lower urinary tract obstruction: a systematic review. Ultrasound Obstet. Gynecol. 37 629-637 (2011)

61. Sananes, N. et al. Urological fistulas after fetal cystoscopic laser ablation of posterior urethral valves: surgical technical aspects. Ultrasound Obstet. Gynecol. 45, 183-189 (2015).

62. Vinit, N. et al. Fetal cystoscopy and vesicoamniotic shunting in lower urinary tract obstruction: long-term outcome and current technical limitations. Fetal Diagn. Ther. 47, 74-83 (2020).

63. Kilby, M. D. \& Morris, R. K. Fetal therapy for the treatment of congenital bladder neck obstruction Nat. Rev. Urol. 11, 412-419 (2014).

\section{Acknowledgements}

ERKNet is endorsed and sponsored by the European Commission.

\section{Author contributions}

V.C., N.P., A.B., S.D., A.F., J.S., E.W., A.Z. researched data for the article, V.C., N.P., A.B., E.A.D.M., D.D.P., W.F., M.H., V.K., G. Manzoni, M.M., J.S., E.W., E.Y., A.Z., F.S., G. Montini made substantial contribution to the discussion of content, V.C. wrote the article, V.C., N.P., S.D., D.D.P., A.F., W.F., M.H., V.K., M.L., G. Manzoni, M.M., G. Mosiello, J.S., J.V.W. E.W. E.Y., A.Z., F.S., G. Montini reviewed/edited the manuscript before submission.

\section{Competing interests}

The authors declare no competing interests.

Peer review information

Nature Reviews Urology thanks M. Farrugia, C. S. Cooper, M. Kilby and K. Pringle for their contribution to the peer review of this work.

Publisher's note

Springer Nature remains neutral with regard to jurisdictional claims in published maps and institutional affiliations.

(c) Springer Nature Limited 2022 